\title{
Reliable detection of Bacillus anthracis, Francisella tularensis and Yersinia pestis by using multiplex qPCR including internal controls for nucleic acid extraction and amplification
}

\author{
Ingmar Janse*, Raditijo A Hamidjaja, Jasper M Bok, Bart J van Rotterdam
}

\begin{abstract}
Background: Several pathogens could seriously affect public health if not recognized timely. To reduce the impact of such highly pathogenic micro-organisms, rapid and accurate diagnostic tools are needed for their detection in various samples, including environmental samples.

Results: Multiplex real-time PCRs were designed for rapid and reliable detection of three major pathogens that have the potential to cause high morbidity and mortality in humans: B. anthracis, F. tularensis and Y. pestis. The developed assays detect three pathogen-specific targets, including at least one chromosomal target, and one target from B. thuringiensis which is used as an internal control for nucleic acid extraction from refractory spores as well as successful DNA amplification. Validation of the PCRs showed a high analytical sensitivity, specificity and coverage of diverse pathogen strains.

Conclusions: The multiplex qPCR assays that were developed allow the rapid detection of 3 pathogen-specific targets simultaneously, without compromising sensitivity. The application of $B$. thuringiensis spores as internal controls further reduces false negative results. This ensures highly reliable detection, while template consumption and laboratory effort are kept at a minimum
\end{abstract}

\section{Background}

A group of diverse pathogens has the potential to cause high morbidity and mortality in humans -especially if carried by aerosols- even though they do not pose a major threat to public health under normal circumstances. The most menacing bacterial pathogens of this group are Bacillus anthracis, Francisella tularensis and Yersinia pestis, and these organisms are listed as category A biothreat agents (classification of the CDC, USA, http://www.bt.cdc.gov/agent/agentlist-category.asp) because of the potential danger of their deliberate release. Exposure to aerosolized B. anthracis spores and $F$. tularensis can lead to inhalational anthrax and tularemia. Y. pestis may cause pneumonic plague, which,

\footnotetext{
* Correspondence: Ingmar.Janse@rivm.nl
National Institute for Public Health and the Environment, Laboratory for

* Correspondence: Ingmar.Janse@rivm.nl
National Institute for Public Health and the Environment, Laboratory for Zoonoses and Environmental Microbiology, Anthonie van Leeuwenhoeklaan 9, 3721 MA Bilthoven, The Netherlands
}

(c) 2010 Janse et al; licensee BioMed Central Ltd. This is an Open Access article distributed under the terms of the Creative Commons Attribution License (http://creativecommons.org/licenses/by/2.0), which permits unrestricted use, distribution, and reproduction in any medium, provided the original work is properly cited.

\section{son to person.}

To reduce the public health impact of such highly pathogenic micro-organisms, rapid and accurate diagrecognition of disease agents will enable appropriate treatment of exposed individuals which will be critical to their survival, and the spread of disease can be reduced by taking appropriate public health measures. Classical identification involves culturing suspect pathogens, but although culturing can be very sensitive, these methods are time consuming, not very specific, involve extensive biosafety measures and some organisms simply resist cultivation. Real-time qPCR methods for the detection of pathogens can be equally or more sensitive, and can also provide higher speed and specificity. Also, molecular methods require only preparatory handling of samples under biosafety conditions and can be easily scaled-up, which is important for speeding up nostic tools for their detection are needed. Timely 
investigations and control of disease progression in outbreak situations. Despite these manifold advantages, detection of DNA does not yield information about the presence of viable organisms.

Multiplexing qPCR detection offers several advantages, including reduction of sample volume and handling time (reducing the analysis time, cost and opportunities for lab contamination). Also, false-negative results can be reduced through co-amplification of internal controls in each sample, and using multiple redundant genetic markers for each organism reduces the chance that strain variants are missed. Amplification of multiple signature sequences per organism will also reduce falsepositive results in complex samples. False positives can be an issue if detection relies on single targets when analyzing environmental samples, due to the presence of homologous sequences in related organisms or unknown sources $[1,2]$. Therefore, it is essential to validate the qPCR using multiple strains, including of closely related organisms.

The selection of suitable signature sequences is an essential requirement for reliable PCR assays. The suitability of signature sequences may be based on their function, e.g. detection of virulence factors supplies important information. But also the stability of their association with the pathogen is of importance. For instance, virulent $B$. anthracis can be recognized by its virulence plasmids pXO1 and pXO2 [3] which contain genes that confer toxin production and capsule synthesis activities, respectively. However, there are also chromosomally encoded factors that are important for the full virulence of $B$. anthracis [4]. Also, recent studies have shown the occurrence of a plasmid homologous to pXO1 in a pathogenic $B$. cereus strain [5] as well as genes homologous to genes on $\mathrm{pXO} 2$ in environmental Bacillus isolates [2]. This underscores the importance of inclusion of a chromosomal signature for B. anthracis in addition to the detection of plasmid genes. Similarly, virulent $Y$. pestis possesses 3 plasmids involved in virulence, but these plasmids are not stable and pathogenic $Y$. pestis lacking any of these plasmids exists [6].

Several reports have described real-time PCR assays for the detection of B. anthracis [7-10], Y. pestis $[6,11,12]$ and $F$. tularensis [13-15]. Some assays were designed in multiplex format, but only few of these included internal controls for DNA amplification $[10,16]$ and none included an internal control for successful DNA extraction. Here, we report the highly reliable and sensitive detection of these three pathogens that we achieved by developing multiplex qPCRs for 3 organism-specific markers and 1 internal control. By using a $B$. thuringiensis gene as internal control, it is possible to use the highly refractory spores of this near relative of B. anthracis as a control for both DNA extraction and
qPCR amplification. The assays were extensively validated and were used on different real-time PCR platforms. The multiplex qPCRs are being applied in screening protocols and our setup allows straightforward expansion of the detection capabilities by inclusion of additional pathogens.

\section{Results}

\section{Design of multiplex hydrolysis probe assays}

A selection of signature sequences for the specific detection and partial characterization of B. anthracis, $F$. tularensis and $Y$. pestis was based on previous reports [4-6,8,11-14,17], and sequence data accessible via public databases (NCBI/EMBL). Additional sequences were obtained from $s s p$ genes from a number of strains from the Bacillus cereus group in our culture collection and from the cry 1 gene from $B$. thuringiensis strain ATCC 29730. Based on signature sequence alignments, regions were identified that were shared by all strain variants and sufficiently different from homologous sequences to design selective oligonucleotides for multiplex real-time qPCR assays (see Table 1).

In order to achieve a reliable as well as rapid method for the detection of $B$. anthracis, $Y$. pestis and F. tularensis, the cry 1 gene from $B$. thuringiensis was included in the multiplex qPCR assays. Inclusion of this gene permitted the development of B. thuringiensis spores as internal control for DNA extraction as well as amplification. The amount of spores that must be added to the samples before DNA extraction to obtain the desired $\mathrm{Cq}$ value was determined from serial dilutions of the spores.

\section{Specificity and coverage of strain diversity}

A DNA panel from the Bacterial and Eukaryal species listed in Additional file 1 Table S1 was used to validate the specificity of the developed real-time qPCR assays. The pathogen-specific targets showed no cross-reactivity and very near relatives could be differentiated as evidenced by the absence of amplification from various members of the Bacillus cereus group, Yersinia pseudotuberculosis, Y. enterocolitica and Francisella philomiragia. From the latter species, one out of the four strains that were tested showed very weak amplification of the multicopy sequence ISFtu2, but none of the strains showed amplification of the $F$. tularensis signature sequence fop $A$. The assays detected all available strains from the targeted organisms. Nevertheless, the genomic marker ypo393 was amplified from only one strain (NCTC 10329) out of four from a $Y$. pestis cluster from Nairobi. Additional information about the pathogens could be derived from the detection of particular plasmid combinations in the $B$. anthracis and $Y$. pestis assays, and from the detection of the $p d p D$ gene [14] in the F. tularensis assay. This was confirmed by the 
Table 1 Primers and probes for multiplex qPCR

\begin{tabular}{|c|c|c|c|c|}
\hline Organism & Target & Oligo function & Oligo name & Sequence $5^{\prime}-3^{\prime a}$ \\
\hline \multirow[t]{9}{*}{ B. anthracis } & sspE & Forward primer & spEpri_f & CGACTGAAACAAATGTACAAGCAGTA \\
\hline & & Reverse primer & spEpri_r & CGTCTGTTTCAGTTGCAAATTCTG \\
\hline & & Probe & Tqpro_spE & FAM-TGCTAGCATTCAAAGCACAAATGCTAGTT-BHQ1 \\
\hline & cya & Forward primer & cyapri_f & AGGTAGATTTATAGAAAAAAACATTACGGG \\
\hline & & Reverse primer & cyapri_r & GCTGACGTAGGGATGGTATT \\
\hline & & Probe & Tqpro_cya & JOE-CCACTCAATATAAGCTITATTACCAGGAGC-BHQ1 \\
\hline & $c a p B$ & Forward primer & caBpri2_f & AGCAAATGTTGGAGTGATTGTAAATG \\
\hline & & Reverse primer & caBpri2_r & AAAGTAATCCAAGTATTCACTITCAATAG \\
\hline & & Probe & Tqpro_caB & CFR590-AGGTCCCATAACATCCATATGATCTTCTAA-BHQ2 \\
\hline \multirow[t]{9}{*}{ F. tularensis } & fopA & Forward primer & foApri_f & GCGCTITGACTAACAAGGACA \\
\hline & & Reverse primer & foApri_r & CCAGCACCTGATGGAGAGTT \\
\hline & & Probe & Tqpro_foA & FAM-TGGCCAGTGGTACTTAGGTGTAGATGCTA-BHQ1 \\
\hline & ISFtu2 & Forward primer & isfpri2_f & CAAGCAATTGGTAGATCAGTTGG \\
\hline & & Reverse primer & isfpri2_r & GACAACAATATTTCTATTGGATTACCTAAA \\
\hline & & Probe & Tqpro_isf & JOE-ACCACTAAAATCCATGCTATGACTGATG-BHQ1 \\
\hline & $p d p D$ & Forward primer & pdDpri_f & TCAATGGCTCAGAGACATCAATTAAAAGAA \\
\hline & & Reverse primer & pdDpri_r & CACAGCTCCAAGAGTACTATTTCC \\
\hline & & Probe & Tqpro_pdD & CFR590-ACCAAATCAAAATCCTGCTGAGCAGA-BHQ2 \\
\hline \multirow[t]{9}{*}{ Y. pestis } & уро393 & Forward primer & yp93pri_f & AGATAGTGTGACTGGTCTTGTTTCA \\
\hline & & Reverse primer & yp93pri_r & AGATGCAGATTGTATTGTAAACAATGAC \\
\hline & & Probe & Tqpro_yp93 & FAM-ACTTCCTGATATATTGGAAATCTTCTTCTC-BHQ1 \\
\hline & caf1 & Forward primer & cafpri_f & CCAGCCCGCATCACT \\
\hline & & Reverse primer & cafpri_r & ATCTGTAAAGTTAACAGATGTGCTAGT \\
\hline & & Probe & Tqpro_caf & JOE-AGCGTACCAACAAGTAATTCTGTATCGATG-BHQ1 \\
\hline & pla & Forward primer & plapri_f & ATGAGAGATCTTACTTTCCGTGAGAA \\
\hline & & Reverse primer & plapri_r & GACTTTGGCATTAGGTGTGACATA \\
\hline & & Probe & Tqpro_pla & CFR590-TCCGGCTCACGTTATTATGGTACCG-BHQ2 \\
\hline \multirow[t]{3}{*}{ B. thuringiensis } & cry1 & Forward primer & crypri_f & GCAACTATGAGTAGTGGGAGTAATTTAC \\
\hline & & Reverse primer & crypri_r & TTCATTGCCTGAATTGAAGACATGAG \\
\hline & & Probe & Tqpro_cry & Cy5-ACGTAAATACACT-BHQ2-TGATCCATTTGAAAAG-P \\
\hline
\end{tabular}

a CFR590 = CALFluor Red 590, BHQ = Black Hole quencher, $\mathrm{P}=$ phosporylation

anticipated absence of the $p d p D$ gene in the $16 F$. tularensis holarctica strains we tested. However, the probe designed for $p d p D$ detection could not discriminate between subspecies tularensis and novicida. Based on the available sequences from $F$. tularensis mediasiatica, amplification of $p d p D$ from this subspecies will occur as well, however, we did not have genomic materials to verify this. Amplification of the pla target from Rattus rattus DNA was unexpected and seemed to indicate cross-reactivity. To confirm pla amplification we investigated DNA from 10 rats, including 3 from the related species Rattus norvegicus (Additional file 1 Table S1). Sequencing of the amplification product from these samples revealed the presence of a pla gene highly similar to that of $Y$. pestis ( $99 \%$ identity), while no sequences with any homology to these sequences were encountered in the published rat genome. Therefore, the amplification does not invalidate our assay but highlights the fact that the pla gene alone is not a sufficient diagnostic marker for the presence of $Y$. pestis. The internal control gene cry 1 was amplified from several Bacillus cultures in addition to $B$. thuringiensis.

Efficiency, dynamic range, precision and detection limit Ten-fold independent serial dilutions from purified target amplicons (PCR products containing target sequences) were used to generate calibration curves and calculate PCR amplification efficiencies. As shown in Table 2 efficiencies for the different targets ranged between $94.5 \%$ and $94.8 \%$ for B. anthracis, between $95.9 \%$ and $98.2 \%$ for F. tularensis and between $93.1 \%$ and $93.2 \%$ for $Y$. pestis. The efficiency for amplification of the internal control target $c r y 1$ varied slightly between the assays and was near that of the organism-specific targets. The reaction was linear over 6 orders of magnitude, from $1.5 \cdot 10^{2}$ to $1.5 \cdot 10^{7}$ target copies per reaction (data not shown). 
Table 2 Precision and detection limits of the multiplex PCRs

\begin{tabular}{|c|c|c|c|c|c|}
\hline organism & Target & Efficiency (\%) & $\begin{array}{l}\text { Repeatability } \\
\left(\mathrm{SD} \text { of } \mathrm{C}_{\mathrm{q}}\right)^{\mathrm{a}}\end{array}$ & LOD target amplicons (copies/reaction) ${ }^{\mathbf{b}}$ & LOD gDNA (fg/reaction) ${ }^{b}$ \\
\hline \multirow[t]{3}{*}{ B. anthracis } & sspe & 94.5 & 0.045 & $2.6(1.6-7.5)$ & $22.6(9.9-148.5)$ \\
\hline & cya & 94.7 & 0.057 & $6.5(3.7-19.6)$ & $50.5(19.1-408.3)$ \\
\hline & $c a p B$ & 94.8 & 0.051 & $3.6(2.0-10.7)$ & $15.7(9.9-78.9)$ \\
\hline \multirow[t]{3}{*}{ F. tularensis } & fopA & 98.2 & 0.042 & $7.2(3.5-24.7)$ & $11.8(5.5-66.4)$ \\
\hline & ISFtu2 & 98.1 & 0.075 & $4.1(2.2-12.8)$ & $0.6(0.2-3.4)$ \\
\hline & $p d p D$ & 95.9 & 0.067 & $6.1(3.1-20)$ & $4.2(2.5-25.6)$ \\
\hline \multirow[t]{3}{*}{ Y. pestis } & уро0393 & 93.1 & 0.057 & $1.7(1.2-3.5)$ & $116(59.3-967.2)$ \\
\hline & caf1 & 93.2 & 0.099 & $1.9(1.3-4.1)$ & $43.2(23.9-277.2)$ \\
\hline & pla & 93.1 & 0.047 & $3.6(2.2-8.9)$ & $29.6(13.5-191.9)$ \\
\hline B. thuringiensis & cryl & $94.6 / 95 / 92.9^{c}$ & $0.047 / 0.055 / 0.057^{c}$ & $\mathrm{ND}$ & $\mathrm{ND}$ \\
\hline
\end{tabular}

${ }^{a}$ Values represent the average from the standard deviations calculated at 5 different dilutions from 4 replicate Cqs measurements.

${ }^{b}$ Values displayed represent the lowest DNA concentration at which $95 \%$ of the positive samples are detected, as calculated by using probit analysis. Shown between brackets are the $95 \%$ confidence limits of the calculated LODs.

${ }^{c} B$. thuringiensis internal control added to B. anthracis, F. tularensis and $Y$. pestis, respectively

ND $=$ not determined

The precision of the different qPCR assays was calculated from 4 replicates of 5 independent dilutions. Mean Cq values and standard deviations (SD) were calculated from each dilution. As shown in Table 2 there is a high repeatability for the different targets, with SDs around $0.05 \mathrm{Cq}$. Only at very low concentrations (high Cq values) near the limit of detection, the SD exceeded 1 Cq (data not shown).

To determine the analytical sensitivity for each single target, dilutions of target amplicons near the detection limit were measured by using the developed assays. The analytical sensitivity for genomic DNA was calculated from dilutions of purified genomic DNA from selected pathogens. The fraction of positive reactions in replicate dilutions were scored and a probit analysis was used to calculate the limit of detection (LOD), which is the lowest concentration at which $95 \%$ of positive samples are detected. The LOD for single targets could be expressed as copy numbers as the target amplicons were of known size. Table 2 shows LODs of below 10 copies for the various targets. For genomic DNA, LODs based on the most sensitive target were for B. anthracis $15.7 \mathrm{fg}$, for $F$. tularensis $0.6 \mathrm{fg}$ and for $Y$. pestis $29.6 \mathrm{fg}$.

\section{Co-amplification targets in multiplex assay}

Large concentration differences between DNA templates in a multiplex PCR may lead to competition for reaction components and impaired amplification of the rarer templates. Divergence of target concentrations could originate from different copy numbers of the targets within the pathogen genome, or from differences between the numbers of organisms that are detected simultaneously. Although there is limited copy number variation for the selected targets, multicopy sequences such as insertion sequences and plasmid genes could outnumber single-copy targets by a factor of more than $200[3,18]$. To exclude an inhibitory effect of the dominant amplification product in the multiplex reaction, dilution series of the high copy number targets (cya, pla and ISFtu2) were made in the presence of a constant and low concentration of the other targets from that organism, and measured by the multiplex qPCRs (Figure $1 \mathrm{~A}-\mathrm{C}$ ). No inhibitory effect (increasing Cq) was observed, even if the excess target considerably exceeded the maximum ratio that could be anticipated.

Significant concentration differences are possible between the pathogen specific targets and the internal control target, as these organisms could be mixed in very different quantities. Inhibition of the internal control (IC) by excess pathogen DNA is not a problem as the function of the IC is to exclude false negative results (a positive pathogen signal makes an additional IC signal irrelevant). In contrast, it is essential that inhibition of pathogen targets by the internal control is prevented. To determine the boundaries within which IC B. thuringiensis DNA could be added to pathogen DNA without interfering with the detection of low pathogen concentrations, a dilution series of the IC target amplicon (cry1 gene) was made in the presence of a constant and low concentration of pathogen targets and measured by the multiplex qPCRs. As shown in Figure 1D-F, the amplification of 20 copies of pathogen targets was inhibited (increasing Cq) if more than 200 copies of the internal control target were present for $B$. anthracis or more than 2000 copies for $Y$. pestis and F. tularensis. Moreover, rare targets were still detectable at much higher excess ratios of internal control, even though at higher Cq values. 

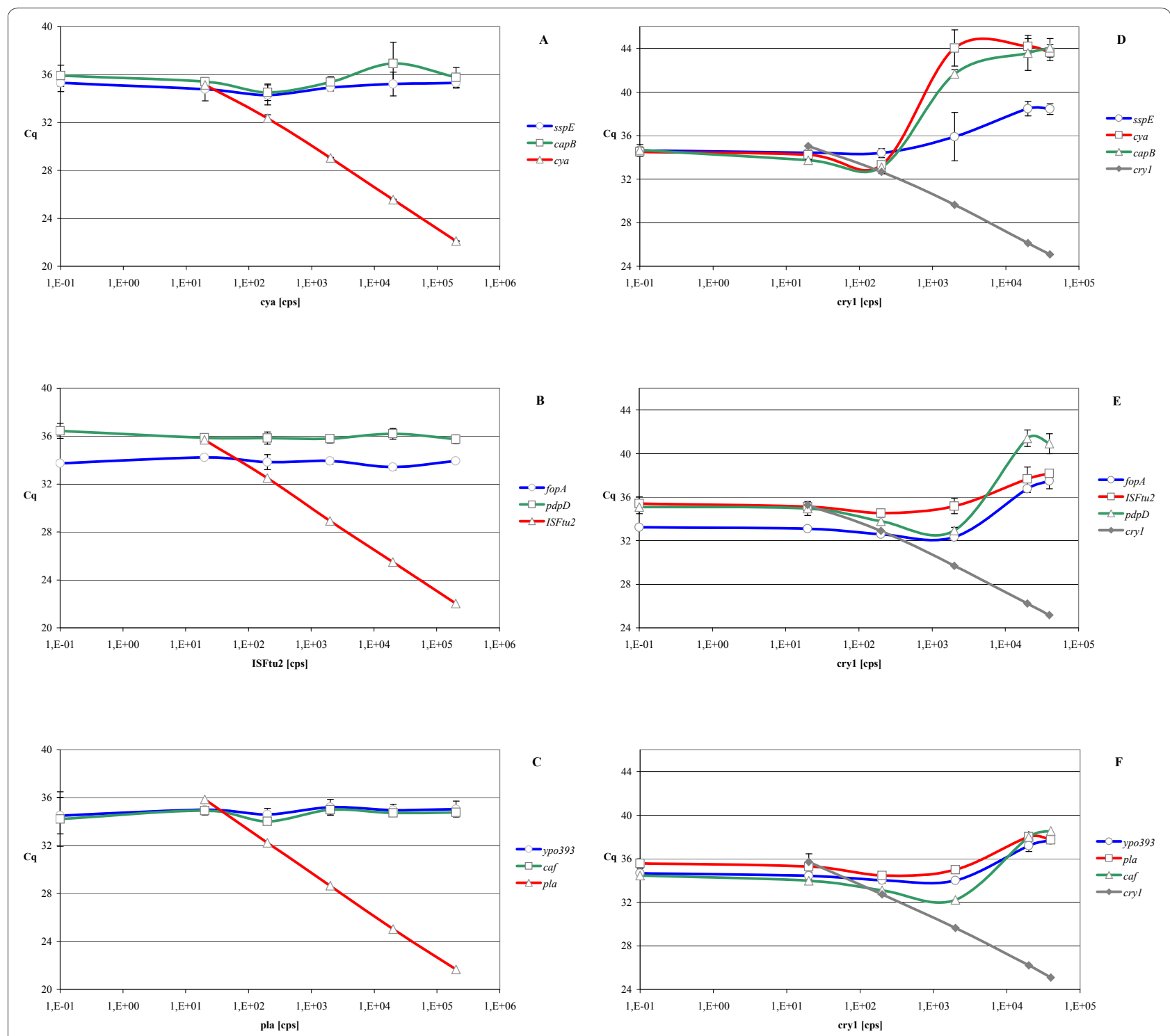

Figure 1 Effect of increasing concentration differences between targets in multiplex qPCR reactions. Dilution series of multicopy targets (A-C) or internal control target cry $\mathbf{1}(\mathrm{D}-\mathrm{F})$ were made in the presence of the other targets detected in each qPCR at a constant concentration near the detection limit. Triplicate multiplex qPCR measurements were performed and mean Cq values with 95\% confidence limits are shown for each target.

\section{Discussion}

Multiplexing and the reduction of false negative and false positive results

In this report, we describe the development of multiplex qPCRs for the rapid and reliable detection of $B$. anthracis, F. tularensis and $Y$. pestis. The assays include a signature sequence from $B$. thuringiensis which allows the use of its spores as combined internal control for both DNA extraction and subsequent DNA amplification. As Bacillus spores are among the most resistant of microbial structures, DNA extraction from such spores can be considered to be a reliable indicator for successful DNA extraction from other microbes. Application of internal controls is especially important when measuring environmental samples because these tend to contain various sorts of PCR inhibitors. The internal control helps preventing false negative results, which are further reduced by the sensitivity of the methods and by the recognition of multiple signatures per organism. Multiplexing reduces the chance that the pathogen escapes detection due to modification or loss of plasmids or genes (natural or by manipulation).

Multiple diagnostic signatures per pathogen will also help reducing false positive detection, which is 
particularly important in complex (environmental) samples which may contain homologous genes of yet uncharacterized origin[1,2]. The genera Bacillus, Francisella, and Yersinia each include species ranging from nonpathogenic environmental species, through symbionts and facultative pathogens, to highly virulent human and animal pathogens. Comparative genomic sequencing and typing studies have indicated that the sequence similarity and gene composition of species having very different lifestyles can be very high $[1,19-21]$ Also, bacterial genomes are dynamic and non-target organisms could acquire diagnostic sequences by lateral gene transfer, especially if present on plasmids [22]. An additional reason for including multiple targets is that for $B$. anthracis and $Y$. pestis, a full picture of virulence requires the detection of several markers. Although virulent $Y$. pestis usually contains three plasmids, strains deficient in one or more plasmids may cause fatal infections [6].

Assays relying on one signature sequence for the detection of a pathogen $[10,23,24]$, suffer from the constraints mentioned above, especially when analyzing environmental samples [1]. For instance, $Y$. pestis subgroup Pestoides lacks the plasminogen coagulase ( $p l a)$ gene [25] that is used as the major and sometimes only target for the detection of $Y$. pestis $[23,26]$. On the other hand, we found that the pla gene may yield false positive results in certain matrices (unpublished). In addition to relying on multiple targets, false positives are further reduced by the high specificity of the developed assays for the selected targets, which was confirmed by in silico and in vitro validations.

\section{Selected targets}

Inclusion of chromosomal markers in addition to virulence plasmids is important due to the occurrence of $B$. anthracis and $Y$. pestis strains lacking virulence plasmids. These strains, as well as yet uncharacterized closely related environmental species, share genomic traits that could lead to misidentification. Fully virulent $B$. anthracis strains possess plasmids pXO1 and pXO2. However, the detection of plasmids only, as for instance commercial kits do, cannot detect plasmid-deficient $B$. anthracis strains such as Sterne and CDC 1014. Moreover, B. cereus strains carrying plasmid highly similar to those of B. anthracis (B. cereus G9241) are not correctly identified. Several chromosomal markers have been used for the detection of $B$. anthracis (e.g. BA813, rpoB, gyrA, gyrB, saspB, plcR, BA5345, BA5510), but only recently a locus was described for qPCR that did not yield any false positive results from closely related Bacillus [27]. We have developed an alternative chromosomal signature sequence $(s s p E)$ for use in real-time PCR. This marker has previously been used for specific detection of $B$. anthracis, but differentiation required melting curve analysis [8]. By selecting highly discriminating positions for primers and hydrolysis probe, we achieved specific detection without post-PCR analysis. For $Y$. pestis, it is equally important to detect chromosomal sequences in addition to its plasmids, as plasmid-deficient virulent $Y$. pestis has been described [6]. Most of the chromosomal targets that have been described previously did not differentiate $Y$. pestis from closely related $Y$. pseudotuberculosis or $Y$. enterocolitica [12]. The chromosomal signature sequence we developed for $Y$. pestis detection was based on a previous study employing comparative genome hybridization to identify chromosomal regions specific for $Y$. pestis [17]. We selected a different region than the ypo2088 target which was used by these authors and later by Matero et al. [16], because examination of published genomes revealed that strain $Y$. pestis antiqua (accession \# CP000308) does not possess this region. Although ypo339 was present in all $20 \mathrm{Y}$. pestis sequences currently publicly available, 3 out of 4 isolates from the Nairobi cluster appeared to lack this signature sequence. Hence, although ypo393 is a reliable signature sequence for most $Y$. pestis, strains lacking this sequence do exist. Our results illustrate that even if signature sequences selected for diagnostic purposes are based on a considerable amount of sequences available from genomes and sequence databases, uncharacterized strain variants may exist or new variants may arise that do not posses a particular target sequence. Conversely, amplification of the cry 1 gene from some Bacillus strains other than $B$. thuringiensis was not anticipated as these strains were not known to contain the plasmids carrying cry genes or homologues. Since it concerned related, spore-forming Bacillus strains, these could also be used as internal controls. The primary focus of our assays was the sensitive and specific detection of the selected pathogens, minimizing false negative and false positive results. Strain differentiation was considered to be of only secondary interest. For F. tularensis, sensitive detection requires detection of the multicopy sequence ISFtu2. The targeted tranposase can also be present in F. philomiragia, but strain ATCC 225017 for instance, has only one copy with mismatches in the probe and reverse primer. This explains the very low cross-reactivity with the four strains we investigated. Nevertheless, specific detection of the species $F$. tularensis was confirmed by additional detection of the fop $A$ gene $[13,15]$. Further subspecies information could be obtained from the $p d p D$ target, which is known to be absent in subspecies holarctica (type B) [14] and was indeed not detected in the 16 strains we tested. With all targets positive, subsequent research is warranted however, as presence of this gene could also imply presence of the subspecies novicida and mediasiatica [28]. Subspecies mediasiatica 
is, similar to subspecies holarctica, a considerable public health threat although both species are less pathogenic compared to subspecies tularensis. Subspecies novicida is less pathogenic than the other subspecies and has been involved in only a limited number of human cases.

\section{Sensitivity}

The analytical sensitivity for detection of the different signature sequences is very high (Table 2). Hence, the presence of only a few genomes should enable detection of the organisms of interest at $95 \%$ probability, especially when based on multicopy signature sequences. For $F$. tularensis this means that only 0.3 genomic equivalents (GE) were sufficient for the detection, considering a genome size of 1.9 megabases. For B. anthracis and $Y$. pestis, reliable estimates of GE could not be made due to the variable and sometimes significant contribution of plasmids to the total amount of DNA measured [3,18]. But, using approximate plasmid copy numbers, a detection limit of $4 \mathrm{GE}$ for B. anthracis and $6 \mathrm{GE}$ for $Y$. pestis can be calculated. The LODs were similar or lower than those reported previously $[13,14]$ and lower than those of other multiplex assays for these pathogens [12]. A correlation between the copy numbers of the targeted genes and the LOD for genomic DNA can be expected. For $F$. tularensis gDNA, the LOD was indeed highest based on the detection of the single-copy fop $A$ target, lower when based on the 2-copy $p d p D$ and lowest when based on the approximately 20-copy ISFtu2 (Table 2). Also for $Y$. pestis, an inverse correlation between gDNA LOD and expected target copy number was observed (Table 2). Nevertheless, a more pronounced difference would be expected based on the high relative abundance of pla carrying plasmids that has been reported [18]. Probably, the gDNA we used contained fewer plasmids, as was supported by a $\mathrm{Cq}$ difference between the chromosomal target and pla of only approximately 2 (data not shown). For B. anthracis, the LOD of gDNA was highest when based on the detection of the pXO1 plasmid marker cya, while high copy numbers for the pXO1 plasmid carrying this gene have been reported [3]. This discrepancy could be due to the gDNA preparation we used for calculating LODs. Although Coker et al. reported relative amounts of pXO1 and pXO2 of respectively 11.5 and 1.6, for the same strain we used (B. anthracis Vollum), variation in pXO plasmid copy numbers could also result from the growth phase at which DNA was harvested [3]. Our data correspond better to the lower plasmid copy numbers reported by other authors $[29,30]$. Nevertheless, all reports agree that pXO1 is present in multiple copies. The relatively high LOD for gDNA detection based on cya can probably partly be explained by a low amplification efficiency near the detection limit as the LOD for the detection of cya target amplicons is also relatively high (Table 2).

\section{Internal control}

As was shown in Figure 1 the cry1 gene from B. thuringiensis spores can be used as internal control without affecting sensitive detection of the pathogens of interest. However, addition of more than 200 copies of $c r y 1$ per reaction lead to a $\mathrm{Cq}$ increase for the detection of the $B$. anthracis plasmid targets. For diagnostic purposes, we use a spore suspension that yields a $\mathrm{Cq}$ value between 32 and 35 for the detection of cry 1 to prevent any interference with the detection of pathogen DNA. The amount of spores that needs to be added to yield this $\mathrm{Cq}$ should be determined for each new batch as it will vary with each new spore stock, and the DNA extraction protocol used. The observed inhibition highlights that multiplex qPCR can be problematic if it is used for the detection of mixed pathogens present in different quantities as amplification of targets from a dominant organism could inhibit the detection of an uncommon pathogen. Assays for the detection of single targets from multiple pathogens simultaneously, such as that described for B. anthracis, F. tularensis and $Y$. pestis detection [23], should therefore be carefully evaluated for this inhibition effect.

\section{Environmental testing}

Application of the multiplex qPCR assays directly on human specimens or environmental samples could save time and prevent loss of DNA during extraction. However, we use the assays only after a DNA extraction protocol, in order to prevent unanticipated inhibition by diverse matrices. Our laboratory has compared several commercially available DNA extraction kits for use in a BSL3 facility, and selected one that combined efficient DNA extraction with ease-of-use and applicability in the restricted BSL3 environment. We have been using the developed qPCRs for the analysis of samples suspected for the presence of these pathogens with $B$. thuringiensis spores added before DNA extraction under BSL3 biosafety conditions. Hundreds of samples containing all sorts of solid materials and liquids have been analyzed without yielding false positive readings.

\section{Conclusion}

The multiplex qPCR assays that were developed for $B$. anthracis, $F$. tularensis and $Y$. pestis allow the rapid detection of 3 pathogen-specific targets simultaneously without compromising sensitivity. Together with the application of an internal control for both DNA extraction and DNA amplification, this assures highly reliable detection, while template consumption and laboratory 
effort is kept at a minimum. These considerations are particularly advantageous in the context of biothreat samples which may be used for additional tests and for surge capacity during an outbreak. The detection of multiple targets decreases the chance of false-positive and false-negative results and provides additional information about virulence.

\section{Methods}

\section{Selection signature sequences}

An initial selection of potential signature sequences for specific detection of B. anthracis, F. tularensis and $Y$. pestis was based on previous reports and on the availability of sequences through public databases (NCBI/ EMBL). The selection was based on functional and on technical criteria. Since 4 reporter dyes can be reliably differentiated by using qPCR instruments, and 1 channel was reserved for the internal control, we selected 3 signature sequences per organism. If possible, signature sequences included virulence genes since these are significant diagnostic markers. Such virulence genes are often located on plasmids. Besides plasmid-encoded targets, at least one chromosomal target was included to account for plasmid transfer and loss. Plasmids may be transferred between closely related species of Bacillus or Yersinia [8]. Plasmids can be cured from B. anthracis [31] and $Y$. pestis [6], and virulent plasmid-deficient $Y$. pestis strains occur in nature [6]. Also, near-neighbor species carrying closely-related plasmids [5] should be distinguished from $B$. anthracis. Finally, although $B$. anthracis has two plasmids that are required for virulence, there are also chromosomally encoded factors that are important for the full virulence [4]. If available, a multicopy sequence was included to enhance sensitivity. Unique targets present only in the organism of interest were preferred over targets differentiating homologues in related species only by sequence differences. Finally, an important consideration for the selection of targets was the quality of sequence information available from the public databases. This sequence quality concerned the number of sequences, their length and their coverage of strain diversity. For each potential target sequence, representative sequences were retrieved from NCBI/EMBL. BLAST searches were then performed to retrieve all homologous sequences from nucleotide and bacterial genome databases. All available sequences were aligned and consensus sequences were created using an accept level of $100 \%$ (to make sure the consensus sequence displayed all sequence variation). For B. anthracis, genes were selected on the multicopy virulence plasmids pXO1 and pXO2, and on the chromosome. The consensus alignment from the toxin gene cya included this gene from the homologous pBCXO1 plasmid which is present in a virulent $B$. cereus strain
[5]. The chromosomal target for B. anthracis, the spore structural gene $\operatorname{ss} E$, is not a unique gene as it is present in all Bacillus. Nevertheless, this sequence was selected since the sequence differences between $B$. anthracis and other species within the closely related $B$. cereus group were sufficient for designing highly selective oligonucleotides. Also, the presence of a substantial number of sequence entries in the databases ( $>200$ ) enabled a reliable consideration of the sequence diversity of $B$. cereus group isolates. For $F$. tularensis, the multicopy insertion sequence ISFtu2 was selected for the detection of $F$. tularensis. Cross reaction with other Francisella species such as F. philomiragia could not be ruled out based on the available sequences, and a region of the outer membrane protein gene fop $A$ was selected for the specific detection of all subspecies from the species F. tularensis. A specific location in the pdpD gene, which is absent from $F$. tularensis subspecies holarctica, was selected for the design of a probe for the detection of F. tularensis subspecies tularensis (type A) [14]. For $Y$. pestis, genes were selected on Y. pestis specific virulence plasmids pPCP1 and pMT1. The plasmid pCD1 was not used as it is shared by other pathogenic Yersinia species. A chromosomal sequence of unknown function that had been identified using comparative genome hybridization [17] was selected as $Y$. pestis specific chromosomal target.

Spores of B. thuringiensis were used as internal control, not only for DNA amplification but also for successful DNA extraction. This member of the B. cereus group is closely related to $B$. anthracis and forms similar spores, while it contains species-specific plasmids. The $B$. thuringiensis plasmid gene encoding insecticidal crystal proteins (cry genes) was used as the signature sequence for the detection of DNA released from this organism's spores.

\section{Sequence analysis tools, bioinformatics software}

Sequences retrieved from NCBI/EMBL were organized and aligned using the software package Kodon (Applied Maths, Ghent, Belgium). Comprehensive sequence alignments were made by performing BLAST searches from the selected targets to make sure all available sequence homologues were included in the alignments. Oligonucleotides for multiplex qPCR assays and for conventional PCR assays were designed using the software package Visual Oligonucleotide Modeling Platform version 6 (DNA software Inc. Ann Arbor, USA). The design strategy for multiplex qPCR assays was as follows. First, a hydrolysis probe and primer set were designed for the $B$. thuringiensis internal control. Then, for each selected signature sequence a hydrolysis probe was designed, followed by the design of the corresponding primer set. A different strategy was chosen for the 
B. anthracis assay, because its chromosomal target $s s p E$ has homologues in other Bacillus, notably the internal control B. thuringiensis. To make sure that detection of $B$. anthracis $\operatorname{ssp} E$ was highly selective, the exact positions of probe and primers were guided based on visual inspection of the alignment. Probe and primers were located in regions with mismatches between Bacillus species (notably between $B$. thuringiensis and B. anthracis), and the primers were designed such that mismatch positions were located at their highly discriminating 3'ends. Oligonucleotides that were calculated by the design software were first checked against the consensus alignment to exclude designs not covering all sequence variants, and were then evaluated using the simulation module of Visual OMP. All oligonucleotides designed were validated in silico by using BLAST searches in general and microbial genomes databases (NCBI/EMBL).

\section{Sequencing}

Sequences were obtained from the cry 1 gene from $B$. thuringiensis strain ATCC 29730 and from the sspE gene from all $B$. anthracis strains in our culture collection, B. thuringiensis ATCC 29730 and B. cereus strains WSBC 10583, 10619, 10766, 10483, 10572, 10705, 10770 and 10865 (Additional file 1 Table S1). In addition, the pla gene was sequenced from DNA extracted from muscle tissue derived from a dissected specimen of Rattus rattus. Primers used for sequencing are displayed in Additional file 2 Table S2. PCR products were purified by using ExoSAP-IT (USB, Cleveland, USA) and DNA sequencing reactions were performed in both directions using BigDyeTerminator v3.1 (Applied Biosystems, Nieuwerkerk a/d IJssel, the Netherlands) on a 48-capillary 3730 DNA Analyzer sequencer (Applied Biosystems, Nieuwerkerk a/d IJssel, the Netherlands). Accession numbers: HQ222846 to HQ222861 and HQ606074.

\section{PCR and real-time qPCR}

Oligonucleotides were synthesized by Biolegio (Biolegio, Nijmegen, the Netherlands). Conventional PCR was used to produce amplicons from signature sequences. Amplification was carried out using the HotStar Taq Master Mix Kit (Qiagen, Westburg, the Netherlands) and $400 \mathrm{nM}$ primers in a total reaction volume of $50 \mu \mathrm{l}$. Primer sets were designed using Visual OMP software (Additional file 2 Table S2). Thermocycling conditions were as follows: $95^{\circ} \mathrm{C}$ for $15 \mathrm{~min}, 40$ cycles at $95^{\circ} \mathrm{C}$ for $30 \mathrm{sec}, 55^{\circ} \mathrm{C}$ for $30 \mathrm{sec}$ and $72^{\circ} \mathrm{C}$ for $30 \mathrm{sec}$, followed by a final step at $72^{\circ} \mathrm{C}$ for $7 \mathrm{~min}$. Thermocycling reactions were carried out in a Px2 thermal cycler (Thermo Electron Corporation, Breda, the Netherlands).

All qPCR reactions were carried out in a final volume of $20 \mu$ l containing iQ Multiplex Powermix (Bio-Rad, Veenendaal, the Netherlands), $200 \mathrm{nM}$ of each primer and 100-300 nM hydrolysis probes and $3 \mu \mathrm{l}$ of DNA template. Probes concentrations had been optimized to yield minimal spectral overlap between fluorescence level of the reporter dyes for each target in a multiplex assay and were 100, 200, 300 and $300 \mathrm{nM}$ for FAM, JOE, CFR590 and Cy5 labeled probes respectively. The multiplex real-time qPCR assays had been designed for an optimal annealing temperature of $60^{\circ} \mathrm{C}$ and the thermal cycling conditions were as follows: First enzyme activation at $95^{\circ} \mathrm{C}$ for $5 \mathrm{~min}$, followed by amplification and detection by 45 cycles at $95^{\circ} \mathrm{C}$ for $5 \mathrm{sec}$ and $60^{\circ} \mathrm{C}$ for $35 \mathrm{sec}$. Each real-time qPCR experiment included a negative (no template) control. Measurements were carried out on a Lightcycler 480 (Roche, Almere, the Netherlands). An iQ5 (Bio-Rad) instrument was used for routine screening purposes. Analyses were performed on the instruments software: LightCycler 480 Software release 1.5.0. SP3 and iQ5 Optical Systems Software version 2.0. Cq values were calculated using the second derivative method on the LightCycler and the Base Line Subtracted Curve Fit method on the iQ5. Color compensations were carried out on both instruments as follows. PCR amplifications were performed using single primer-probe sets for each reporter dye and under identical reaction conditions as during multiplex amplification. The PCR reactions thus produced contained single dyes in relevant concentrations and these were used for color compensation runs according to the manufacturers' guidelines. Verification of PCR product sizes were carried out on the 2100 Bioanalyzer instrument (Agilent Technologies, Eindhoven, the Netherlands) using the DNA 1000 kit.

\section{Bacterial isolates and genomic DNA preparation}

The detection limits and specificities of the assays were evaluated using genomic materials from the bacterial strains and other sources displayed in Additional file 1 Table S1. The pathogen panel included (besides a variety of Eukaryal organisms): 8 B. anthracis strains and 31 near relatives $(22 \mathrm{~B}$. cereus, $5 \mathrm{~B}$. thuringiensis and $4 \mathrm{~B}$. mycoides), 21 F. tularensis strains (16 subspecies holarctica, 4 tularensis and 1 novicida) and 4 of the closest related species $F$. philomiragia, $23 Y$. pestis (including Antiqua, Mediaevalis and Orientalis biovars) and 3 strains from the closest relative $Y$. pseudotuberculosis and 7 strains from $Y$. enterocolitica. From most of the $B$. anthracis, F. tularensis and $Y$. pestis strains we only had genomic DNA (lysates) available to verify specificity of our assays. Several strains were available as live cultures in our laboratory and these were used as resource for the production of larger quantities of genomic DNA. $B$. anthracis and $Y$. pestis strains were acquired from the NCTC (National Culture Type Collection, UK) and the Pasteur Institute (France). The Francisella holarctica 
strain was a patient isolate. Other genomic materials were lysates from bacterial cultures provided by other researchers as mentioned in the acknowledgements. Cultivation of these strains was carried out in a BSL3 glove-box. Colonies from B. anthracis, F. tularensis and $Y$. pestis were grown on Columbian sheep blood agar plates and chocolate agar plates. Single colonies were transferred to liquid BHI (Brain Heart Infusion, $27 \mathrm{~g} / \mathrm{L}$ ) medium. After cultures had grown to visible turbidity, $1.4 \mathrm{ml}$ cell culture was centrifuged and the pellet was resuspended in $250 \mu \mathrm{l} \mathrm{TE} \mathrm{pH} \mathrm{8.} \mathrm{Cells} \mathrm{were} \mathrm{incubated}$ for 30 minutes at $100^{\circ} \mathrm{C}$. Lysed cultures were filtered through a $0.22 \mu \mathrm{m}$ sterile Ultrafree-MC spinfilter (Millipore, Amsterdam, the Netherlands) and the filtrate was subsequently transported from the BSL3 facility for handling under normal laboratory conditions. Cultures from non-target bacteria that were used in the specificity panel were obtained from the culture collection at the RIVM. These cultures were cultivated under BSL2 conditions and lysates of these cultures were used for specificity testing.

DNA extraction and purification was carried out by using NucliSens Magnetic Extraction Reagents (bioMérieux, Boxtel, the Netherlands) following the manufacturers instructions. This method performed best with regard to efficiency and ease-of-use when compared to other kits. This comparison was carried out as follows. Dilution series of a mixture of genomic DNA from $B$. anthracis, $Y$. pestis and $F$. tularensis, and spores from $B$. thuringiensis were added to various powders including milk powder, soy powder, silica and maize powder, and DNA was extracted by using the powersoil DNA isolation kit (MO BIO Laboratories, Carlsbad, USA), the ultraclean microbial DNA isolation kit (MO BIO) and the NucliSens Magnetic Extraction Reagents (bioMérieux) according to the manufacturers instructions. The extracts were measured by using the developed qPCR. DNA concentrations were measured using the NanoDrop 1000 spectrophotometer (Thermo Fisher Scientific, Wilmington, USA). DNA samples were stored at $4^{\circ} \mathrm{C}$ for use within 1 week and at $-20^{\circ} \mathrm{C}$ for longer storage.

\section{Spore suspension for use as internal control}

Spore suspensions of $B$. thuringiensis strain ATCC 29730 (var. galleriae Heimpel) were obtained from Raven Biological Laboratories (Omaha, Nebraska, USA). These washed spores were counted by microscopy and then aliquotted and stored at $4^{\circ} \mathrm{C}$. The amount of spores that needs to be added to samples to obtain suitable $\mathrm{Cq}$ values for this internal control must be determined empirically for each stock spore suspension. Ten-fold serial dilutions were made from the spore stock and DNA was extracted from $50 \mu \mathrm{l}$ portions of each dilution by using the Nuclisens Magnetic Extraction Reagents
(bioMérieux). The developed real-time qPCR assays were used to determine the amount of spores required for a Cq value between 32 and 35 .

\section{Limit of detection, efficiency and repeatability}

Characterization of qPCR performance was guided by the MIQE guidelines [32]. The validation was carried out by using genomic DNA as well as purified PCR amplicons including $>100 \mathrm{bp}$ upstream and downstream from the qPCR amplification sites. The latter were used to compose template mixes of desired composition and quantities, while maintaining secondary structures in the primer binding regions. Detection limits (LOD) for genomic DNA were determined by using purified DNA from cultures of B. anthracis strain Vollum, F. tularensis strain tularensis ATCC 6223 and $Y$. pestis strain Harbin. DNA was purified from lysates of these strains. The concentration of purified genomic DNA was measured by using the NanoDrop 1000 spectrophotometer. Serial dilutions of genomic DNA were used to calculate LODs from the proportion of positive qPCRs at each dilution. Four replicates of eight serial dilutions of genomic DNA were measured by qPCR. Based on the results, an additional measurement was performed on 4 replicates of 8 novel serial dilutions. The measurements included at least one dilution with all replicates positive and one with all replicates negative. A probit analysis was performed using SPSS Statistics 18.0.0 to calculate the DNA concentration that could be measured with $95 \%$ probability.

DNA templates for measuring the detection limits from the different signature sequences were amplified from the bacterial strains mentioned above. In addition, the pdpD signature sequence from $\mathrm{F}$. tularensis tularensis was amplified from ATCC 6223. To generate suitable amplicons for testing the different real-time qPCR targets, primers were designed for amplification of a signature sequence with a size of 400-800 bp, extending beyond both ends of the region amplified by the realtime qPCR. Primer sequences are displayed in Additional file 2 Table S2. After amplification, PCR products were purified and the number of DNA copies in amplicon solutions was calculated from their sizes and concentrations. Amplicon dilutions were used to calculate the LOD from the proportions of positive qPCRs at each dilution. First, 5 replicates of 8 dilutions around the estimated detection limit were measured using a mixture of equal amounts of target amplicons. Based on the results, an additional measurement was performed on 10 replicates of 8 novel dilutions. After scoring positive results, a probit analysis was performed to calculate the DNA concentration that could be measured with $95 \%$ probability. 
Efficiency and repeatability were calculated from the log-linear portion of the calibration curve, covering 6 orders of magnitude. The calibration curve was made using amplicon mixtures as templates containing the signature sequences (as described before). Four replicate measurements were obtained from each dilution. For calculation of the repeatability, the lowest template concentration was not used as the standard deviation (SD) near the detection limit was not consistent with those obtained for the other concentrations.

\section{Dynamic range internal control}

To establish a concentration range for the applicability of the internal control, serial dilutions were made of internal control cry 1 target amplicon $\left(0,2 \cdot 10^{1}, 2 \cdot 10^{2}\right.$, $2 \cdot 10^{3}, 2 \cdot 10^{4}, 4 \cdot 10^{4}$ copies per reaction) in the presence of a mixture of the 3 organism specific target amplicons, each at a concentration of 20 copies per reaction. These target amplicon mixtures were amplified in triplicate by using the developed qPCR assays and $\mathrm{Cq}$ values were used to infer possible inhibition of PCR amplification. To investigate inhibitory effects on the amplification of organism-specific targets, triplicate measurements were performed on amplicons of the multicopy targets (cya, pla and ISFtu2) diluted as above in the presence of the 2 other organism-specific target amplicons, each at a concentration of 20 copies per reaction.

\section{Additional material}

Additional file 1: Table S1 - Panel of organisms used for coverage and specificity analysis. This table lists the different strains of the targeted pathogens, their close relatives, and a selection of other Bacteria and Eukarya that were used to validate the specificity of the developed multiplex qPCR assays. Amplification results are given for each signature sequence.

Additional file 2: Table S2 - Primer sequences for conventional PCR. This table displays the primers that were developed for convential PCR. These primers were applied for sequencing and for the production of target amplicons that were used for assay validation.

\begin{abstract}
Acknowledgements
We gratefully acknowledge Horacio Gill from the Centro Nacional de Microbiologia, Instituto de Salud Carlos III, Majadahonda, Spain, Rickart Knuttson and Joakim Ågren from the National Veterinary Institute (SVA), Uppsala, Sweden, the Swedish Defense Research Agency (FOI), Umea, Sweden, Karen Kempsell from the Health Protection Agency (HPA), Porton Down, UK, and Jasper Kieboom from TNO Defense and Safety, Rijswijk, the Netherlands, for providing genomic materials. Frans Reubsaet, Maaike de Vries, Marieke Opsteegh and Chantal Reusken from CIB, RIVM are acknowledged for sharing bacterial cultures and other genomic materials. This work was funded by a SOR strategic research grant from the RIVM.
\end{abstract}

\section{Authors' contributions}

IJ: conceived the study and designed the experiments, performed oligonucleotide designs and statistical analyses, interpreted experimental results and wrote the manuscript; $\mathrm{RAH}$ : participated in the design of the experiments, carried out and interpreted the experimental work, and helped to draft the manuscript; JMB: helped carrying out experiments; BVR: coordinated the work. All authors read and approved the final manuscript.

Received: 25 May 2010 Accepted: 8 December 2010

Published: 8 December 2010

\section{References}

1. Kuske CR, Barns SM, Grow CC, Merrill L, Dunbar J: Environmental survey for four pathogenic bacteria and closely related species using phylogenetic and functional genes. Journal of Forensic Sciences 2006, 51(3):548-558

2. Luna VA, King DS, Peak KK, Reeves F, Heberlein-Larson L, Veguilla W, Heller L, Duncan KE, Cannons AC, Amuso P, Cattani J: Bacillus anthracis virulent plasmid pX02 genes found in large plasmids of two other Bacillus species. Journal of Clinical Microbiology 2006, 44(7):2367-2377.

3. Coker PR, Smith KL, Fellows PF, Rybachuck G, Kousoulas KG, HughJones ME: Bacillus anthracis virulence in Guinea pigs vaccinated with anthrax vaccine adsorbed is linked to plasmid quantities and clonality. Journal of Clinical Microbiology 2003, 41(3):1212-1218.

4. Koehler TM: Bacillus anthracis genetics and virulence gene regulation. Current Topics in Microbioogy and Immunology 2002, 271:143-164.

5. Hoffmaster AR, Ravel J, Rasko DA, Chapman GD, Chute MD, Marston CK, De BK, Sacchi CT, Fitzgerald C, Mayer LW, Maiden MCJ, Priest FG, Barker M, Jiang LX, Cer RZ, Rilstone J, Peterson JN, Weyant RS, Galloway RS, Read TD, Popovic T, Fraser CM: Identification of anthrax toxin genes in a Bacillus cereus associated with an illness resembling inhalation anthrax. Proceedings of the National Academy of Sciences of the United States of America 2004, 101(22):8449-8454.

6. Tomaso H, Reisinger EC, Al Dahouk S, Frangoulidis D, Rakin A, Landt O, Neubauer $\mathrm{H}$ : Rapid detection of Yersinia pestis with multiplex real-time PCR assays using fluorescent hybridisation probes. FEMS Immunology and Medical Microbiology 2003, 38(2):117-126.

7. Moser MJ, Christensen DR, Norwood D, Prudent JR: Multiplexed detection of anthrax-related toxin genes. Journal of Molecular Diagnostics 2006, 8(1):89-96.

8. Kim K, Seo J, Wheeler K, Park C, Kim D, Park S, Kim W, Chung SI, Leighton T: Rapid genotypic detection of Bacillus anthracis and the Bacillus cereus group by multiplex real-time PCR melting curve analysis. FEMS Immunology and Medical Microbiology 2005, 43(2):301-310.

9. Bell CA, Uhl JR, Hadfield TL, David JC, Meyer RF, Smith TF, Cockerill FR: Detection of Bacillus anthracis DNA by LightCycler PCR. Journal of Clinical Microbiology 2002, 40(8):2897-2902.

10. Panning M, Kramme S, Petersen N, Drosten C: High throughput screening for spores and vegetative forms of pathogenic $B$. anthracis by an internally controlled real-time PCR assay with automated DNA preparation. Medical Microbiology and Immunology 2007, 196(1):41-50.

11. Woron AM, Nazarian EJ, Egan C, McDonough KA, Cirino NM, Limberger RJ, Musser KA: Development and evaluation of a 4-target multiplex real-time polymerase chain reaction assay for the detection and characterization of Yersinia pestis. Diagnostic Microbiology and Infectious Disease 2006, 56(3):261-268.

12. Stewart A, Satterfield B, Cohen M, O'Neill K, Robison R: A quadruplex realtime PCR assay for the detection of Yersinia pestis and its plasmids. Journal of Medical Microbiology 2008, 57(3):324-331.

13. Versage JL, Severin DDM, Chu MC, Petersen JM: Development of a multitarget real-time TaqMan PCR assay for enhanced detection of Francisella tularensis in complex specimens. Journal of Clinical Microbiology 2003, 41(12):5492-5499.

14. Tomaso H, Scholz HC, Neubauer H, Al Dahouk S, Seibold E, Landt O, Forsman M, Splettstoesser WD: Real-time PCR using hybridization probes for the rapid and specific identification of Francisella tularensis subspecies tularensis. Molecular and Cellular Probes 2007, 21(1):12-16.

15. Fujita O, Tatsumi M, Tanabayashi K, Yamada A: Development of a real-time PCR assay for detection and quantification of Francisella tularensis. Japanese Journal of Infectious Diseases 2006, 59(1):46-51.

16. Matero P, Pasanen T, Laukkanen R, Tissari P, Tarkka E, Vaara M, Skurnik M: Real-time multiplex PCR assay for detection of Yersinia pestis and Yersinia pseudotuberculosis. APMIS 2009, 117(1):34-44.

17. Zhou DS, Han YP, Dai EH, Pei DC, Song YJ, Zhai JH, Du ZM, Wang J, Guo ZB, Yang RF: Identification of signature genes for rapid and specific 
characterization of Yersinia pestis. Microbiology and Immunology 2004, 48(4):263-269.

18. Parkhill J, Wren BW, Thomson NR, Titball RW, Holden MT, Prentice MB, Sebaihia M, James KD, Churcher C, Mungall KL, Baker S, Basham D, Bentley SD, Brooks K, Cerdeno-Tarraga AM, Chillingworth T, Cronin A, Davies RM, Davis P, Dougan G, Feltwell T, Hamlin N, Holroyd S, Jagels K, Karlyshev AV, Leather S, Moule S, Oyston PC, Quail M, Rutherford K, et al: Genome sequence of Yersinia pestis, the causative agent of plague. Nature 2001, 413(6855):523-527.

19. Chain PS, Carniel E, Larimer FW, Lamerdin J, Stoutland PO, Regala WM, Georgescu AM, Vergez LM, Land ML, Motin VL, Brubaker RR, Fowler J, Hinnebusch J, Marceau M, Medigue C, Simonet M, Chenal-Francisque V, Souza B, Dacheux D, Elliott JM, Derbise A, Hauser $\sqcup$, Garcia E: Insights into the evolution of Yersinia pestis through whole-genome comparison with Yersinia pseudotuberculosis. Proceedings of the Naional Academy of Sciences USA 2004, 101(38):13826-13831.

20. Rasko DA, Ravel J, Okstad OA, Helgason E, Cer RZ, Jiang L, Shores KA, Fouts DE, Tourasse NJ, Angiuoli SV, Kolonay J, Nelson WC, Kolsto AB, Fraser CM, Read TD: The genome sequence of Bacillus cereus ATCC 10987 reveals metabolic adaptations and a large plasmid related to Bacillus anthracis pXO1. Nucleic Acids Research 2004, 32(3):977-988.

21. Read TD, Peterson SN, Tourasse N, Baillie LW, Paulsen IT, Nelson KE, Tettelin H, Fouts DE, Eisen JA, Gill SR, Holtzapple EK, Okstad OA, Helgason E, Rilstone J, Wu M, Kolonay JF, Beanan MJ, Dodson RJ, Brinkac LM, Gwinn M, DeBoy RT, Madpu R, Daugherty SC, Durkin AS, Haft DH, Nelson WC, Peterson JD, Pop M, Khouri HM, Radune D, et al: The genome sequence of Bacillus anthracis Ames and comparison to closely related bacteria. Nature 2003, 423(6935):81-86.

22. Ochman $\mathrm{H}$, Lawrence JG, Groisman EA: Lateral gene transfer and the nature of bacterial innovation. Nature 2000, 405(6784):299-304.

23. Skottman T, Piiparinen H, Hyytiainen H, Myllys V, Skurnik M, Nikkari S: Simultaneous real-time PCR detection of Bacillus anthracis, Francisella tularensis and Yersinia pestis. European Journal of Clinical Microbiology and Infectious Diseases 2007, 26(3):207-211.

24. Tomioka K, Peredelchuk M, Zhu XY, Arena R, Volokhov D, Selvapandiyan A, Stabler K, Melliquist Riemenschneider J, Chizhikov V, Kaplan G, Nakhasi H, Duncan R: A multiplex polymerase chain reaction microarray assay to detect bioterror pathogens in blood. Journal of Molecular Diagnostics 2005, 7(4):486-494.

25. Worsham PL, Roy C: Pestoides F, a Yersinia pestis strain lacking plasminogen activator, is virulent by the aerosol route. Advances in Experimental Medicine and Biology 2003, 529:129-131.

26. Loiez C, Herwegh S, Wallet F, Armand S, Guinet F, Courcol RJ: Detection of Yersinia pestis in sputum by real-time PCR. Journal of Clinical Microbiology 2003, 41(10):4873-4875.

27. Antwerpen MH, Zimmermann P, Bewley K, Frangoulidis D, Meyer H: Realtime PCR system targeting a chromosomal marker specific for Bacillus anthracis. Molecular and Cellular Probes 2008, 22(5-6):313-315.

28. Mitchell $\mathrm{J}$, , Chatwell N, Christensen D, Diaper H, Minogue TD, Parsons TM, Walker B, Weller SA: Development of real-time PCR assays for the specific detection of Francisella tularensis ssp. tularensis, holarctica and mediaasiatica. Molecular and Cellular Probes 2010, 24(2):72-76.

29. Read TD, Salzberg SL, Pop M, Shumway M, Umayam L, Jiang LX, Holtzapple E, Busch JD, Smith KL, Schupp JM, Solomon D, Keim P, Fraser CM: Comparative genome sequencing for discovery of novel polymorphisms in Bacillus anthracis. Science 2002, 296(5575):2028-2033.

30. Almeida JL, Harper B, Cole KD: Bacillus anthracis spore suspensions: determination of stability and comparison of enumeration techniques. Journal of Applied Microbiology 2008, 104(5):1442-1448.

31. Turnbull PC, Hutson RA, Ward MJ, Jones MN, Quinn CP, Finnie NJ, Duggleby CJ, Kramer JM, Melling J: Bacillus anthracis but not always anthrax. Journal of Applied Bacteriology 1992, 72(1):21-28.

32. Bustin SA, Benes V, Garson JA, Hellemans J, Huggett J, Kubista M, Mueller R, Nolan T, Pfaffl MW, Shipley GL, Vandesompele J, Wittwer CT: The MIQE guidelines: minimum information for publication of quantitative realtime PCR experiments. Clinical Chemistry 2009, 55(4):611-622.

doi:10.1186/1471-2180-10-314

Cite this article as: Janse et al:: Reliable detection of Bacillus anthracis, Francisella tularensis and Yersinia pestis by using multiplex QPCR including internal controls for nucleic acid extraction and amplification. BMC Microbiology 2010 10:314.

\section{Submit your next manuscript to BioMed Central and take full advantage of:}

- Convenient online submission

- Thorough peer review

- No space constraints or color figure charges

- Immediate publication on acceptance

- Inclusion in PubMed, CAS, Scopus and Google Scholar

- Research which is freely available for redistribution

Submit your manuscript at www.biomedcentral.com/submit
C Biomed Central 